In $10 \%(2 / 20)$ patients the operated ear had a profound loss/dead ear pre-operatively. Audiological outcomes consisting of averaged thresholds at $0.5,1,2$ $\& 4 \mathrm{kHz}$ were available for $15 / 18$ of the remaining patients. Mean change in air conduction thresholds was $0 \mathrm{db}$ (median 0, range $-25-+25$ ). Mean change in bone conduction thresholds was $-2 \mathrm{db}$ (median -2 , range $-16-+15)$.

Conclusions: Mastoid cavity obliteration in our experience has been associated with excellent outcomes in terms of dry-ear rate and recurrence rate at median follow up of 15 months.

doi:10.1017/S0022215116001638

Basic and translational research in cholesteatoma and ear surgery (N633)

\section{ID: 633.1}

Cholesteatoma among school-age children and adults - hearing screening program and surgical possibilites

\section{Presenting Author: Piotr Skarzynski}

Piotr Skarzynski ${ }^{1}$, Krzysztof Kochanek ${ }^{2}$, Henryk Skarzynski ${ }^{2}$

${ }^{1}$ Institute of Physiology and Pathology of Hearing, ${ }^{2}$ World Hearing Center, Institute of Physiology and Pathology of Hearing,

Kajetany/ Warsaw, Poland

\section{Learning Objectives:}

Introduction: Restoration of hearing in patients with hearing impairment due to cholesteatoma (and CWU or CWD surgery) with lack of the ossicles, after modified radical operations could be done with direct stimulation of the round window membrane or bone conductive solutions.

Congenital cholesteatoma may occur in different age groups. It can be located in many sites including the cerebellopontine angle, the inner ear, the mastoid, the petrosus apex, the middle ear, the tympanic membrane, the external auditory canal. From the clinical point of view it is very important to detect it as early as possible. Hearing screening in school-age children was performed in Poland and other countries from 2008 to 2015. The program was focused on children at the age of 7-12 years old. The main aim of the program was to detect hearing disorders, which were not observed by the parents or teachers.

The objective of that presentation is analysis hearing results obtained after surgical application of different implants in treatment of hearing impairment patients with chronic inflammation of the middle ear, especially after radical modified operations. Another objective is to present results of cholesteatoma detection in different screening programs among school children around the world.

Material and Methods: The selected group of patients were children and adults with chronic inflammation of the middle ear, after radical modified operations with destruction of the elements of the middle ear - tympanic membrane and ossicles. Group of patient analyzed in tht study was 29312.
We discussed the indications, contraindications and limitations of use of Vibrant Soundbridge in this group of patients.

Results and conclusions: Early detection, especially congenital cholesteatoma, is essential for very good results. There is many possibilities in reconstructive technique for hearing restoration. Each patient should be analyzed individually to different surgical way.

doi:10.1017/S002221511600164X

\section{Basic and translational research in cholesteatoma and ear surgery (N633)}

\section{ID: 633.2}

\section{Imaging follow-up of patients after} cholesteatoma surgery

\section{Presenting Author: Marcin Szymanski \\ Marcin Szymanski}

Medical University of Lublin, Poland

Learning Objectives: There are various techniques of cholesteatoma surgery but all of them carry the risk of residual or recurrent cholesteatoma development. Thus all the patients after cholesteatoma surgery require thorough follow-up and some patients a second look surgery. While open cavity surgery enables otoscopic recognition of cholesteatoma, the use of closed technique, obliteration of mastoid cavity or subtotal petrosectomy reduces the role of clinical examination in follow-up. Imaging modalities including HRCT and non-EP DWI MR is discussed in patients subjected to open or closed techniques, obliteration of the mastoid cavity or subtotal petrosectomy for removal of congenital and acquired cholesteatoma.

doi:10.1017/S0022215116001651

\section{Basic and translational research in cholesteatoma and ear surgery (N633)}

\section{ID: 633.3}

Combined model of intraoperative monitoring of ossiculoplasty efficiency by laser-Doppler vibrometry and auditory evoked potentials

Presenting Author: Krzysztof Morawski

Krzysztof Morawski ${ }^{1}$, Kazimierz Niemczyk ${ }^{1}$, Rafael E Delgado ${ }^{2}$

${ }^{1}$ Medical University of Warsaw, ${ }^{2}$ Department of Biomedical Engineering, University of Miami. Fl, USA

\section{Learning Objectives:}

Objective: To assess utility of combined electrophysiological and laser-Doppler Vibrometry (LDV) technique for intraoperative monitoring (IM) of air-bone gap closure (ABGC) 
during ossiculoplasty. A new strategy of IM of hearing threshold (HT) evaluation was developed by a team of engineers and surgeons on the basis of previously performed researches.

Subjects and Methods: Patients $(\mathrm{n}=25)$ underwent two-stage canal wall-up tympanoplasty due to chronic otitis media with cholestaetoma. During the second look surgery performed 12 months later ossiculoplasty was monitored intraoperatively by LDV and round window electrocochleography (RWECochG). Both measures were performed via an enlarged posterior tympanotomy. LDV and RW-ECochG intraoperative tests recorded simultaneously for the same stimulation set. Intraoperative HT was defined automaticaly in auditory steady state response (ASSR) option as well as prosthesis vibration by LDV. Using both intraoperative techniques various configurations of prosthesis placement were tested. On the basis of the preoperative tonal audiometry and post-ossiculoplasty RW-ECochG \& LDV thresholds a minisoftware calculated an optimal ABGC. Prosthesis moveability tested simultaneously by LDV was showed and correlated with RW-ECochG thresholds.

Results: Postop ABG closure ranged between 15 to $45 \mathrm{~dB}$ HT improvement evaluated intraoperatively correlated with postop ABGC $(r>0.5 ; p<0.05)$. Various prosthesis configurations and placements resulted in measurable changes in the RW-ECochG thresholds. LDV appeared sensitive mostly to prosthesis position changes manifesting by movability improvement at $0.5-$ and $1.0 \mathrm{kHz}$.

Conclusions: RW-ECochG measured in ASSR option was found to be an objective and sensitive technique for IM of HT improvement significantly corresponding with postop ABG-C. LDV showed their usefulness to control prosthesis position changes by confirming better acoustic energy transfer through the reconstructed ossicular chain.

doi:10.1017/S0022215116001663

\section{Basic and translational research in cholesteatoma and ear surgery (N633)}

\section{ID: 633.4}

Preliminary Analysis of Genetic

Alterations in Cholesteatoma

\section{Presenting Author: Krzysztof Szyfter}

Krzysztof Szyfter ${ }^{1}$, Malgorzata Jarmuz-Szymczak ${ }^{2}$, Maciej Giefing ${ }^{2}$, Kinga Bednarek ${ }^{2}$, Wojciech Gawęcki ${ }^{3}$, Witold Szyfter ${ }^{4}$

${ }^{1}$ Institute of Human Genetics, Polish Academy of Sciences, ${ }^{2}$ Institute iof Human Genetics,

Polish Academy of Sciences, ${ }^{3}$ ENT Clinic,

Poznań University of Medical Sciences, ${ }^{4}$ ENT

Clinic, Poznań University of Medical Sciences

\section{Learning Objectives:}

The Clinic is operating annually over 100 choleasteatomas (655 operations in the years 2010 -2015). Because of a common bacterial infection a bacteriologic analysis indicates for Pseudomonas aeruginosa, Proteus mirabilis and Staphylococcus aureus as the most commonly detected in middle ear infection.

Having in mind a literature suggestion of a partial analogy between oncogenesis and cholesteatoma formation and own experience in identification of oncogenes and tumor suppressor genes modulating progression of laryngeal cancer we have undertaken a molecular analysis targeting for an identification of genetic backgroung of cholesteatoma. Array-CGH scanning of a genome indicated for frequent gains and losses of gene copy number in the genome. The results will be further analyzed to identify amplified regions potentially indicating location of oncogenes and homozygous deletions covering loci oftumor suppressor genes involved in cholesteatoma.

Independently a molecular cytogenetic technique was applied to analyze $8 \mathrm{q} 24$ chromosome region to estimate an amplification and potential rearrangement(s) of c-Myc oncogene. Fluorescent in situ Hybridization (FISH) with the use of specific DNA probes (regular fluorescent, break a part) is being applied.

The results will be presented during the meeting.

doi:10.1017/S0022215116001675

\section{Basic and translational research in cholesteatoma and ear surgery (N633)}

\section{ID: 633.5}

Inflammatory pathways in middle ear cholesteatoma

Presenting Author: Ewa Olszewska

Ewa Olszewska ${ }^{1}$, Marek Rogowski ${ }^{2}$, Miroslaw Szczepanski $^{3}$

${ }^{1}$ Medical University of Bialystok, ${ }^{2}$ Medical University of Bialystok, Poland, ${ }^{3}$ Czerniakowski

Hospital, Warsaw, Poland

\section{Learning Objectives:}

Introduction: Middle ear cholesteatoma (MEC), accompanied by chronic inflammatory response is characterized by invasive growth and osteolytic activity.

Aim: Present the cellular and inflammatory pathways in the pathogenesis of cholesteatoma and adjacent tissues.

Material and methods: Congenital, acquired MEC (study groups) and retroauricular skin specimens (control group, CS) were investigated for markers of inflammation using various immunohistochemistry, Western Blot, cell culture and flow cytometry techniques. Studied markers included proliferation and apoptosis of keratinocytes (PCNA, Ki67, p53, p21, APO2.7), angiogenesis and inflammation (TGFa), proteasomal degradation pathway (low-molecular mass polypeptide-7 subunit of the immunoproteasome (LMP7), and selected molecular signalling (the DNA-binding highmobility box 1 (HMGB1) in the protein advanced glycation endproducts (RAGE) axis.

Results: The significantly more intense expression of LMP7 and p21-positive cells was seen in MEC. The LMP7 $(+)$ cells 